\title{
Le point de vue du contrôleur technique
}

\author{
The construction adviser's point of vue
}

\author{
P. BLONDEAU
}

Département Sols et Fondations, SOCOTEC*

Rev. Franç. Géotech. n 58, pp. 15-22 (janvier 1992)

\section{Résumé}

Les sécheresses exceptionnelles des deux dernières années (1989 et 1990) ont provoqué des dégâts très importants dans les constructions récentes et anciennes.

A partir de l'analyse des enquêtes faites en France et de son expérience professionnelle, le contrôleur technique déduit des conclusions pratiques et propose des éléments de réflexion pour de futures recommandations à l'usage des constructeurs.

\section{Abstract}

The unexpected draughts of two last years (1989 and 1991) caused very extensive damages to new and old buildings.

Analysing the results of enquiries made in France and from his own professional experience the construction adviser gives some pratical conclusions to be used in recommendations to building specialists. 


\section{PRÉSENTATION}

La France, qui a la réputation d'un pays au « climat tempéré », nous offre depuis dix ans quelques surprises désagréables.

Citons pour mémoire :

- les tempêtes en Bretagne de novembre 1982 et octobre 1987. Celles du début 1990 dans le Nordouest de la France ;

- les inondations de 1988 avec la catastrophe de Nîmes. La première estimation de la récurrence du phénomène était de mille ans, la dernière est inférieure à cent ans ! Et la toute dernière inondation de décembre 1990 dans les Ardennes de la Meuse, qualifiée "du siècle *;

- les chutes de neige de l'hiver 1986 et celles de décembre dernier dans l'Ain ;

- enfin, et c'est là notre propos, les sécheresses de 1976 et celles des deux dernières années 89 et 90 .

En effet, depuis deux ans, la baisse marquée de la pluviométrie se traduit, entre autres phénomènes, par un assèchement progressif des terrains, surtout argileux ou marneux.

Ces terrains, en se desséchant, se rétractent en entrânant des désordres plus ou moins importants aux ouvrages fondés superficiellement.

Le phénomène est à l'échelon national ; il touche de nombreux départements et ressort de la catégorie des catastrophes naturelles.

Nous allons tenter de faire un premier bilan sur nos informations et connaissances en la matière et essayer de proposer des recommandations pour éviter, ou pour le moins atténuer, les conséquences parfois dramatiques d'un phénomène naturel quî $a$, il faut l'avouer, surpris les géotechniciens.

Il faut bien reconnaitre malheureusement que, ni les règlements (DTU par exemple) ni les études de sols et fondations au sens large du terme n'avaient envisagé ce cas de figure.

On sait estimer des déformations sous charges dans l'étude classige des fondations : c'est l'analyse de l'interaction sol-structure. On connaît les risques de gonflement de certains matériaux argileux (cf. les marnes vertes tristement célèbres de la Région parisienne). On ne s'est pratiquement jamais préoccupé du phénomène de retrait des matériaux argileux par dessication. Les désordres provoqués par la sécheresse de 1976 ont bien fait l'objet d'expertises et d'études particulières mais il n'en a pas été tiré un enseignement général.

\section{RAPPEL DE LA LOI DU 13 JUILLET 1982 POINTS ESSENTIELS}

Cette loi a créé une garantie obligatoire par contrats d'assurance des catastrophes naturelles.

Les effets des catastrophes naturelles sont les dommages matériels ayant pour cause déterminante l'intensité anormale d'un agent naturel lorsque les mesures habituelles à prendre pour prévenir ces dommages n'ont pu empêcher leur survenance ou n'ont pu être prises.
Les dommages matériels qui en découlent sont indemnisés, soit par la « Multirisques Habitation », soit par la « Tout Risque Chantier » (TRC), la « Dommage Ouvrage " étant exclue.

La catastrophe naturelle doit être constatée par Arrêté Interministériel à la suite d'une procédure administrative (avec publication au JO).

De ce bref rappel une série de question-clés en découlent.

Quels sont les critères définissant l'état de catastrophe naturelle "sécheresse "?

Citons :

- critères météorologiques (ensoleillement, durée, seuil) ;

- critères géotechniques (nature du sol, caractéristiques de gonflement ou de retrait).

Dans quelle mesure la sécheresse est-elle la cause déterminante du phénomène?

Certains ouvrages n'étaient-ils pas « aux limtes de l'épure "à la veille de la sécheresse. Cette dernière étant le "révélateur " de leur mauvais état, de leur mauvaise conception ou de leur mauvaise réalisation?

Quelles sont les solutions confortatives?

Doivent-elles être définitives pour se prémunir contre toute nouvelle sécheresse exceptionnelle (il faut bien noter dès à présent que beaucoup d'ouvrages légers où anciens ne permettent pas la "solution maximale " de reprise en sous-œuvre par micropieux)?

Doit-on attendre (et observer) les effets dus au retour à des périodes humides comme actuellement et tenter d'optimiser les solutions confortatives?

Quelles sont les études géotechniques à réaliser pour analyser le "risque de retrait " des sols argileux?

Les essais actuels (in situ en particulier) sont-ils bien adaptés ?

Peut-on définir une méthodologie simple qui soit à la portée de tous les bureaux d'études des sols et fondations?

Quelles seraient les "recommandations " à suivre pour les futurs ouvrages tant au stade conception que réalisation ; en particulier doit-on définir une profondeur " hors sécheresse »?

Il faut préciser dès à présent que la Délégation aux risques majeurs du ministère de l'Environnement a créé trois groupes de travail en novembre 1990 avec les thèmes suivants :

- critères et seuils d'appréciation de l'état de catastrophe naturelle;

- rédaction de " recommandations techniques " traitant des solutions confortatives et préventives ;

- assurance et expertise.

Ces actions doivent être conduites avec diligence et efficacité pour répondre à l'attente des personnes sinistrées.

Par ailleurs, cinq organismes (APSAD, AFAC, CCR, FNB et l'Agence Qualité Construction) ont passé commande au CEBTP d'un recueil de directives précises et pratiques de réparation efficaces et économiques des désordres consécutifs à la sécheresse exceptionnelle des années 1989 et 1990. Ce guide pratique 
devrait être disponible avant la fin 1991. Le CEBTP est aidé dans sa tâche par des membres des groupes de travail de la Délégation aux risques majeurs.

\section{RAPPEL TRÈS SIMPLIFIÉ DU PHÉNOMĖNE PHYSIQUE}

Le retrait des sols de fondation provoqué par la sécheresse n'intéresse pratiquement que les "sols argileux ». Certaines argiles sont plus sensibles au retrait que d'autres, ce sont celles, en particulier, qui contiennent des Montmorillonites ou des Illites.

Une première approche de leur identification est donnée par les limites d'Atterberg. Il faut se méfier des matériaux argileux dont la limite de liquidité est supérieure à 50 et l'indice de plasticité supérieur à 15 .
Ensuite se pose le problème de la mesure et de l'estimation du retrait! (quel essai géotechnique ?)

La sécheresse est définie par deux facteurs, son intensité et sa durée. On retrouve là, et là seulement, l'analogie avec le gel. Il faut préciser tout de suite que les profondeurs dites hors gel n'ont aucun rapport avec les profondeurs atteintes par la sécheresse!

La sécheresse a pour corollaire la pluviométrie. On peut constater, par exemple, qu'en 1989 la pluviométrie n'était que 55 à $85 \%$ par rapport à la normale (fig. 1). Si l'on considère uniquement la pluie efficace, celle qui pénètre dans le sol, on constate durant la même période une baisse générale par rapport à la moyenne (de 0 à $100 \%$ ). Seul le Nord de la France révèle une augmentation (de 1 à 40 \%) (fig. 2).

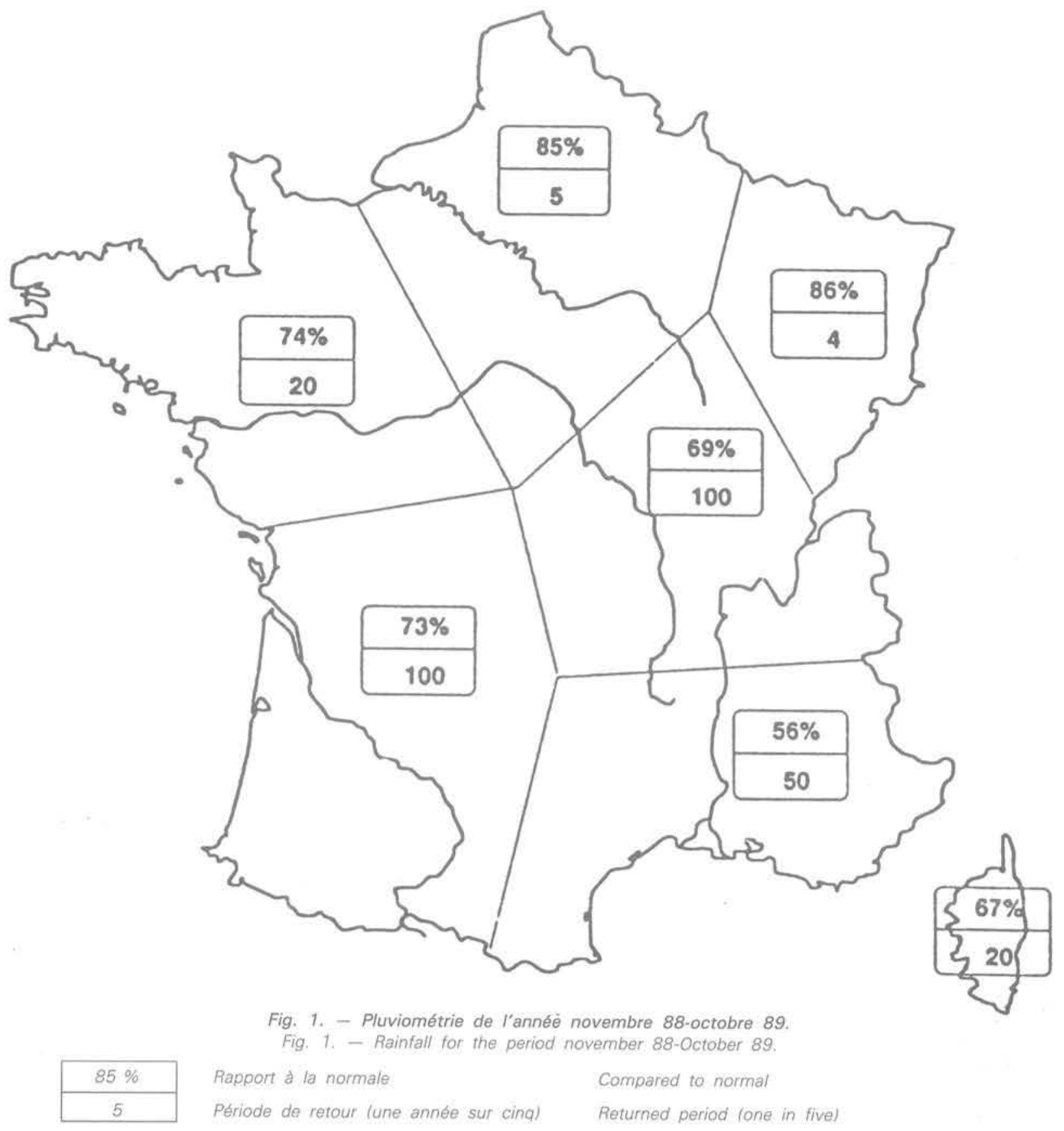




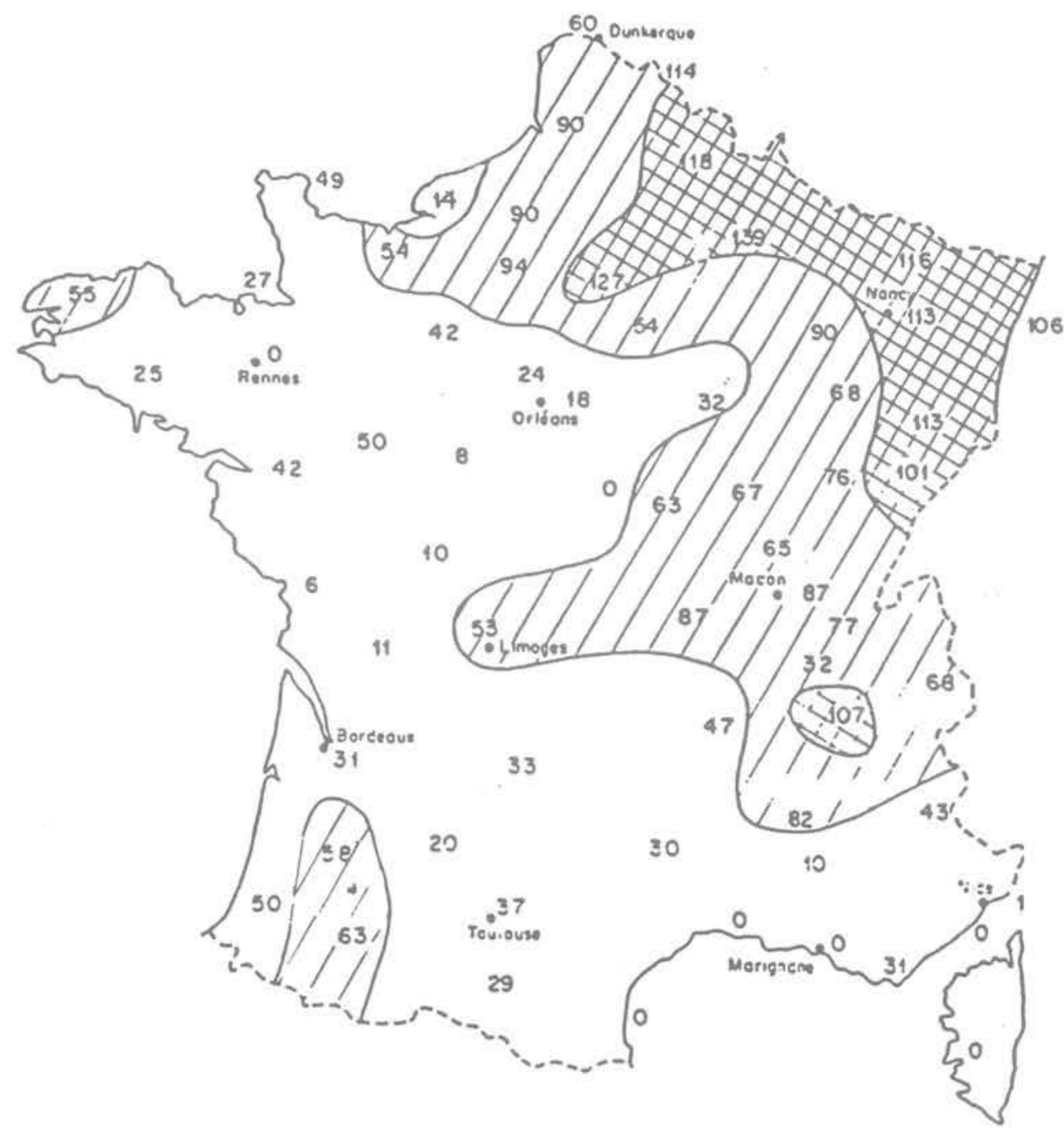

60

Voleur en pour cem se la pluie efficoce de l'onnie por rascart a

la pluie efficoce moyenne

Q7 Pluie efficoce sudertieure dे lo moyenne

DD Pluie efficoce comprise entre la moyenne et $50 \%$ de la moyonne

Pluie efficace inferieure o $50 \%$ de to moyenne

Fig. 2. - Comparaison de la pluie efficace de l'année 1988-89 avec la moyenne de référence.

Fig. 2. - Comparison of "Useful w rainfall for the year 1988-89. Compared with the reference average.

Suivant les régions, et en toute première approximation, la sécheresse aurait fait sentir ses effets sur une profondeur de l'ordre de deux mètres (Nord de la France) à trois mètres (Sud de la France) si l'on excepte l'influence des racines des arbres qui peut dépasser cinq mètres.

Il faut bien noter que la dessication des sols provoque un tassement différentiel pour trois raisons :

- les sols sont hétérogènes même dans l'emprise limitée d'un ouvrage ;

- la répartition des contraintes dans le sol n'est pas homogène ;

- la dessication se propage de l'extérieur vers l'extérieur des fondations par suite du gradient de température.
Il est un acteur bien vivant qui joue le rôle du traitre dans cette scène, c'est l'arbre. La sécheresse a confirmé, s'il en était besoin, le rôle important joué par la végétation et, en particulier, celui de certaines espèces tels les peupliers. Ces derniers, qui sont de gros consommateurs d'eau (200 à 400 1/jour), vont en période de sécheresse puiser leur complément dans l'eau du sol. Lorsque les racines passent au voisinage ou sous les fondations, on conçoit aisément les tassements qui peuvent se produire par assèchement progressif du terrain. Il faut bien avouer que les peupliers n'ont pas attendu la sécheresse pour sévir. Cette dernière n'a fait qu'amplifier leurs méfaits.

Une règle simple, mais peu souvent respectée consiste à implanter des arbres à fort pouvoir d'absorb- 
sion à une distance supérieure à la hauteur de larbre adulte (fig. 3).

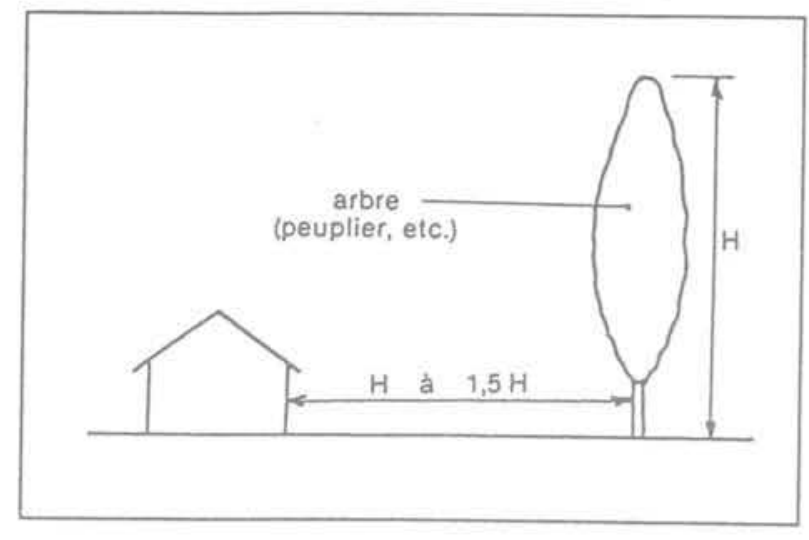

Fig. 3. - Distance minimale entre les bâtiments et les arbres. Fig. 3. - Minimum distance between house and tree.

\section{RÉPARTITION GÉOGRAGPHIQUE ET ANALYSE STATISTIQUE}

Nous avons deux sources d'informations, celle de la Délégation aux risques majeurs et celle de l'Agence qualité construction.

La première nous donne le recensement des départements sinistrés à la mi-novembre 90. C'est ainsi que 216 communes réparties sur 17 départements ont été reconnues sinistrées par Arrêté Interministériel après avis favorable de la commission chargée d'instruire les dossiers transmis par les Préfets. Le département du Nord bat un triste record avec 70 communes*

La seconde nous présente une analyse de la pathologie après dépouillement d'une enquête faite auprès de son réseau d'experts. C'est ainsi qu'elle a donné 305 fiches se rapportant à 356 ouvrages (cf. répartition géographique) (fig. 4 et 5).

Il y a des divergences entre les répartitions des départements sinistrés et celle des expertises. Cela tient au fait que dans la seconde source d'information le "bon vouloir " de l'expert joue un certain rôle.

Mais quelles qu'en soient les disparités, il est possible d'en tirer un certain nombre d'enseignements.

Ainsi les 356 cas recensés se décomposent en :

- maisons individuelles : 340 cas dont 328 constructions nouvelles;

- bâtiment collectif : 1

- autres constructions : 15.

Pour les maisons individuelles on trouve $90 \%$ de rez-de-chaussée.

On voit que les maisons à simple rez-de-chaussée constituent la majorité des bâtiments sinistrés ( $86 \%$ du total des fiches).

Si l'on analyse la nature du plancher bas, on constate que le dallage sur terre-plein vient largement en tête avec 253 cas, soit plus de $70 \%$. Puis viennent les planchers sur vide sanitaire $(\simeq 18 \%)$, les planchers sur sous-sol partiel $(\simeq 6 \%$ ) et les planchers sur sous-sol total $(\simeq 5 \%)$.

L'histogramme des profondeurs de fondations, après correction, montre que la majorité des fondations se situent entre 20 et $80 \mathrm{~cm}$ de profondeur (fig. 6)

On peut donc constater que l'exemple type de l'ouvrage sinistré est la maison individuelle nouvelle à simple rez-de-chaussée avec dallage sur terre-plein. Comme ces maisons représentent environ $35 \%$ du marché, on peut conclure qu'elles présentent le taux de sinistralité le plus élevé.

Dans la quasi-totalité des cas analysés, le sol de fondation est argileux (argile pure, argile calcaire, limon argileux)

Enfin, dans 120 cas sur 356 on signale la présence d'arbres ou d'arbustes au voisinage de la construction (soit $30 \%$ des cas).

Quels sont les désordres constatés? Ce sont des fissures dans les éléments porteurs (murs de façade, pignons) d'amplitude très variable (jusqu'à plusieurs centimètres), souvent en diagonale traduisant des affaissements différentiels des fondations superficielles. On note aussi des fissures horizontales traduisant des décollements des fondations.

Ce sont des affaissements avec ou sans fissuration des dallages. La valeur maximale de tassement signalée est de $12 \mathrm{~cm}$.

Dans $80 \%$ des cas, l'expert a conclu à une reprise en sous-ceuvre, soit en reprise totale $(=30 \%)$, soit en reprise partielle $(\simeq 50 \%)$. Les modes de reprise en sous-œuvre sont les suivants:

- par plots $(\simeq 37 \%)$;

- par micropieux ( $\approx 34 \%$ );

- par semelles continues $(=14 \%$ );

- par injection sous dallage $(\simeq 13 \%)$.

On voit que les deux solutions les plus utilisées sont les plots et les micropieux.

Sans rentrer dans les détails on peut donner les coûts approximatifs suivants :

coût moyen par ouvrage $=100000 \mathrm{~F}(10000 \mathrm{~F}$ sans reprise en sous-cuvre à $111000 \mathrm{~F}$ avec reprise en sous-œuvre).

Ces réparations appellent quelques commentaires de notre part :

- on ne connaît pas encore la « réversibilité » du retrait. Il nous paraît opportun lorsque le cas de figure n'est pas dramatique (risque d'effondrement) d'attendre avant de définir une reprise en sous-œuvre (colmatage des fissures) ;

- la solution de reprise en sous-cuvre est une solution lourde et couteuse qu'il importe d'étudier avec soin (nécessité d'une étude des sols pour dimension-

\footnotetext{
- Plus de 1500 communes sont touchées et plus de 50 départements sont concernés à fín auril 1991.
} 


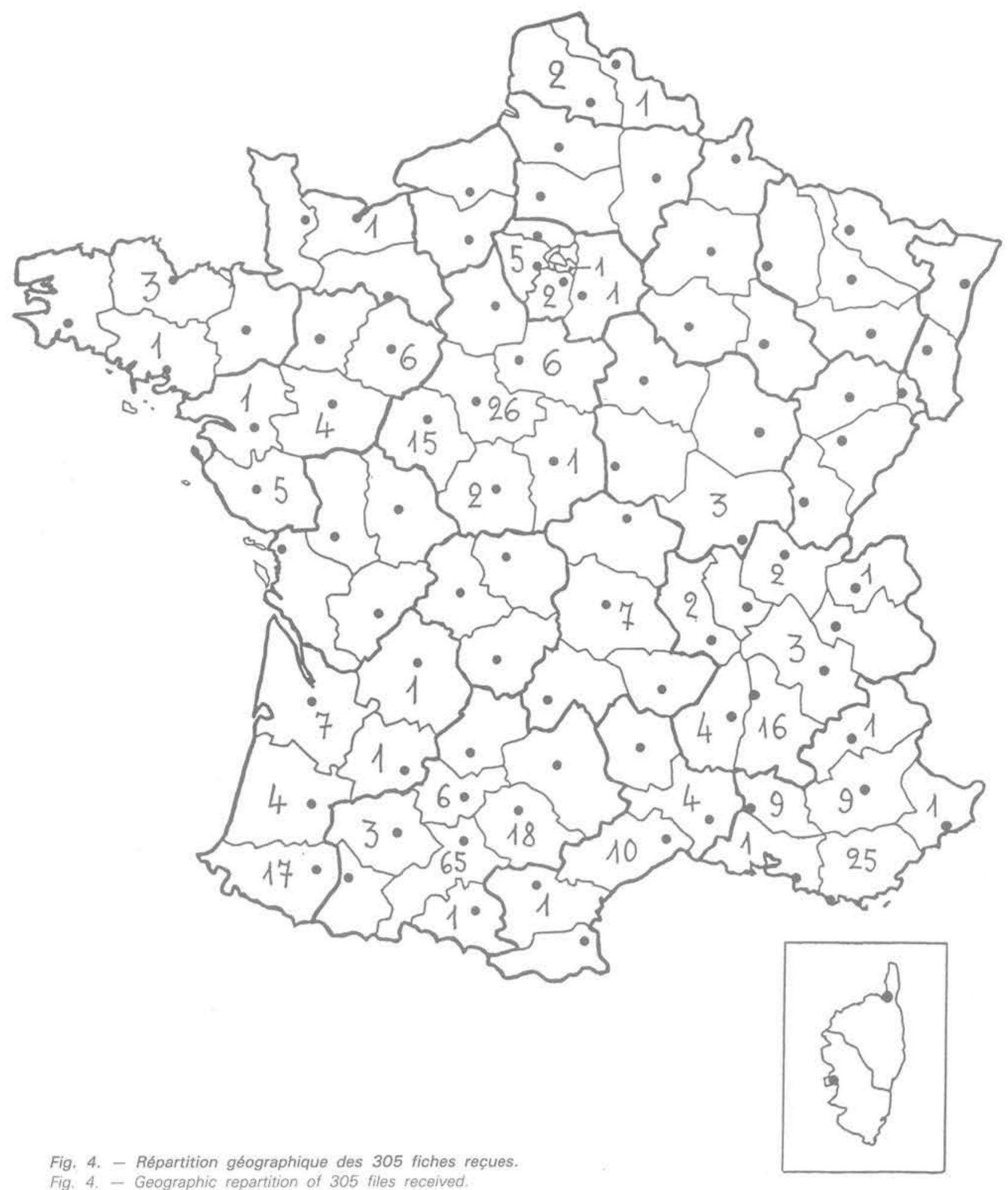

Fig. 4. - Geographic repartition of 305 files received.

ner les plots ou les micropieux). Dans le cas des micropieux, la structure des pavillons ne se prête pas toujours à ce mode de reprise en sous-cuvre : on a pu constater des ruptures de liaison de tête de micropieux. D'une façon générale il est fortement déconseillé de procéder à des reprises en sous-œuvre partielles. La partie traitée crée un point dur qui peut induire dans le temps des désordres dans la partie apparemment saine ;

- on ne signale pas suffisamment le rôle négatif des arbres qu'il faut faire enlever lorsqu'ils sont en cause ! - enfin les injections sous dallage sont délicates à réaliser (absence de vides, défaut de réaction) et peuvent conduire à des désordres. 


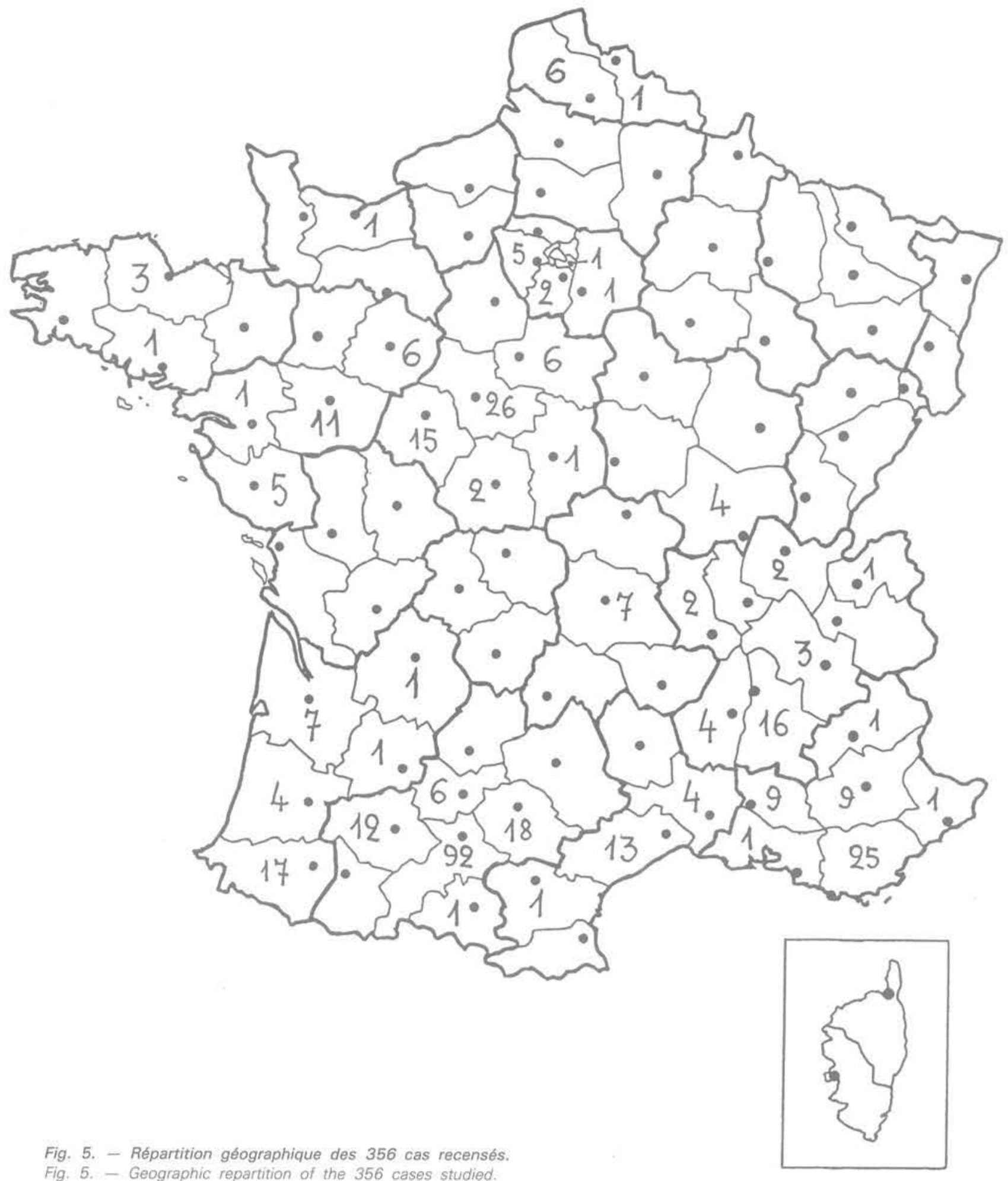

Fig. 5. - Geographic repartition of the 356 cases studied.

\section{Premières conclusions}

Nos réflexions seront très générales, il n'est pas dans notre propos d'imposer des directives, mais simplement de faire quelques rappels pour orienter de futures recommandations.

Au stade de l'étude géotechnique il faut que soient précisées les caractéristiques de gonflement ou de retrait des matériaux argileux suivant un mode opératoire simple et commun à tous les laboratoires.

$\mathrm{Au}$ stade du projet il ne faut pas oublier les règles d'or suivantes :

- maintenir le taux d'humidité naturel du matériau argileux, d'où des notions de protection extérieure et de drainage; 


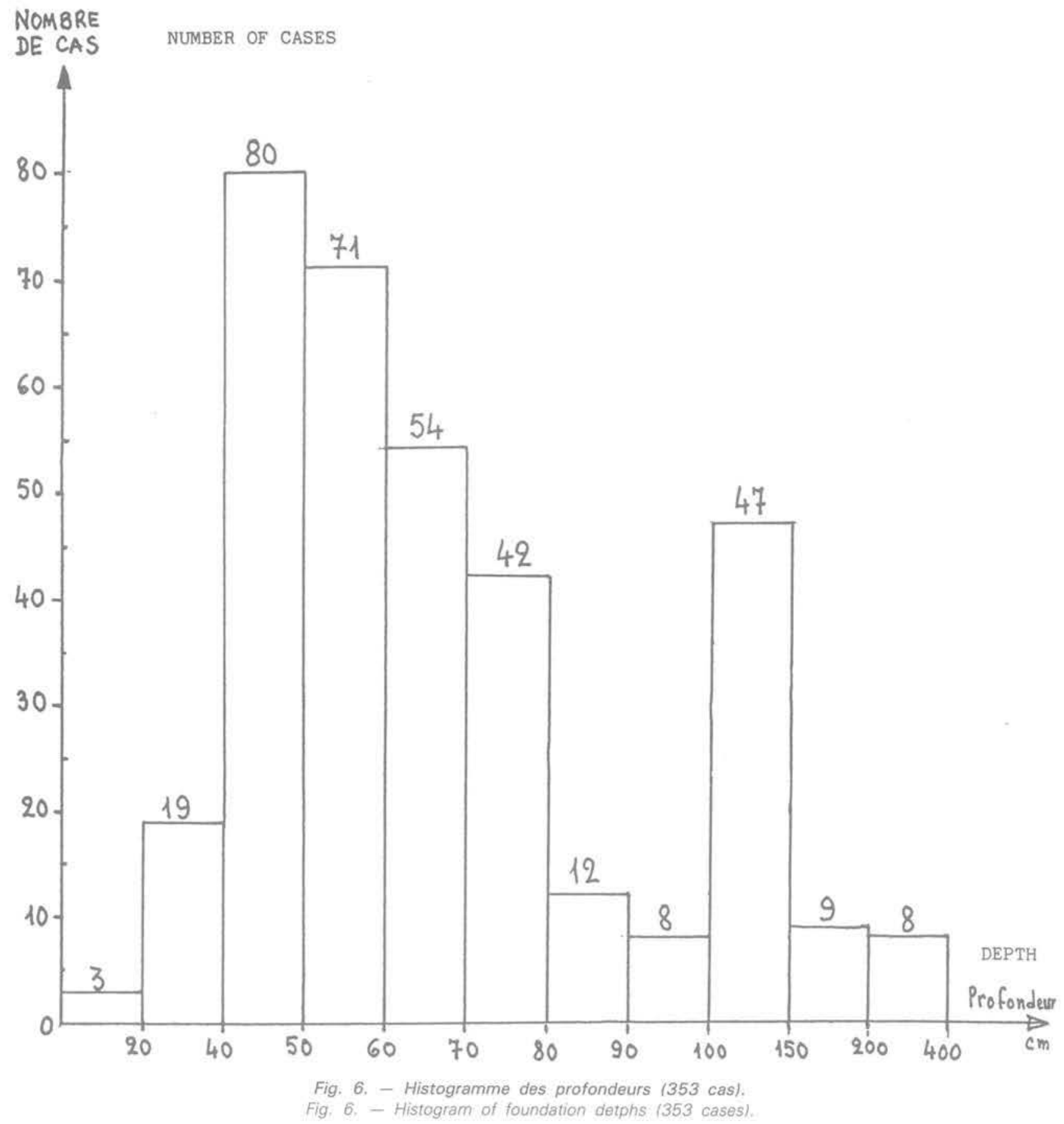

- éviter les dallages sur terre-plein et concevoir des vides sanitaires;

- étudier le meilleur raidissement de louvrage ;

- rechercher un niveau de fondation insensible aux variations de température ou d'humidité : pavillons sur sous-sol; les fondations sur picots constituent une excellente solution économique ;

- proscrire les arbres et arbustes au voisinage;

- ne pas oublier les liaisons souples à l'arrivée ou au départ des canalisations. 